\title{
THE STRONG SUMMABILITY OF DOUBLE FOURIER SERIES
}

\section{HAI-TSIN HSÜ}

1. Introduction. Corresponding to the well known theorem of Fejér-Lebesgue, we have for the double Fourier series the following proposition:

If $f \log ^{+}|f|$ is Lebesgue integrable on the square $(-\pi \leqq x \leqq \pi$, $-\pi \leqq y \leqq \pi)$, then the Fejer mean $\sigma_{m, n}(x, y)$ of $f(x, y)$ tends to $f(x, y)$ almost everywhere as $m$ and $n$ independently increase indefinitely. Moreover, for every increasing function $\phi(t)$ satisfying the conditions

$$
\phi(0)=0, \quad \liminf _{t \rightarrow \infty} \frac{\phi(t)}{t \log t}=0,
$$

there is a function $f(x, y)$ such that $\phi(|f|)$ is integrable and that $\sigma_{m, n}(x, y)$ does not converge almost everywhere. ${ }^{1}$

The latter half of this theorem shows that the analogue, in double Fourier series, of the Fejér-Lebesgue theorem is not a trivial extension of that of a function of a single variable.

The purpose of the present note is to discuss the strong summability ${ }^{2}$ of double Fourier series. A double series $\sum a_{m n}$ is said to be strongly summable with the positive index $k$ if there exists a constant $s$ such that the expression

$$
\frac{1}{(m+1)(n+1)} \sum_{\mu=0}^{m} \sum_{\nu=0}^{n}\left|s_{\mu, \nu}-s\right|^{k} .
$$

has the double limit zero as $m$ and $n$ increase without limit, where

$$
s_{m, n}=\sum_{\mu=0}^{m} \sum_{\nu=0}^{n} a_{\mu \nu} .
$$

It is easily seen from Hölder's equality that the summability says more for larger $k$.

Suppose now that $f(x, y)$ is integrable in the Lebesgue sense over

Received by the editors May 16, 1945.

${ }^{1}$ B. Jessen, J. Marcinkiewicz and A. Zygmund [5]. The first example of a function $f(x, y) \in L$ with Fejér mean divergent everywhere was given by A. Zygmund; see S. Saks [8]. Numbers in brackets refer to the Bibliography at the end of the paper.

${ }^{2}$ A notion first introduced in Fourier series by G. H. Hardy and J. E. Littlewood [1]. For subsequent researches, see Hardy and Littlewood [2, 3], J. Marcinkiewicz [6] and A. Zygmund [12]. 
the square $Q(-\pi,-\pi ; \pi, \pi)$ and is doubly periodic with period $2 \pi$ in each variable. The Fourier series of $f(x, y)$ is

$$
\begin{aligned}
& \sum_{m, n=0}^{\infty} \lambda_{m, n}\left[a_{m, n} \cos m x \cos n y+b_{m, n} \sin m x \cos n y\right. \\
& \left.+c_{m, n} \cos m x \sin n y+d_{m, n} \sin m x \sin n y\right],
\end{aligned}
$$

where

and

$$
\lambda_{m, n}=\left\{\begin{array}{cl}
1 / 4 \text { for } m=n=0 \\
1 / 2 \text { for } m=0, n>0 \text { or } m>0, n=0 \\
0 \text { for } m>0, n>0
\end{array}\right.
$$

$$
a_{m, n}=\frac{1}{\pi^{2}} \int_{Q} \int f(x, y) \cos m x \cos n y d x d y,
$$

and so on.

On writing

$$
\begin{aligned}
4 \phi(u, v) \equiv \phi_{x, y}(u, v)= & f(x+u, y+v)+f(x+u, y-v) \\
& +f(x-u, y+v)+f(x-u, y-v)-4 s,
\end{aligned}
$$

and

$$
\Phi_{x, v}^{(p)}(u, v)=\int_{0}^{u} \int_{0}^{v}|\phi(\xi, \eta)|^{p} d \xi d \eta \quad(p \geqq 1)
$$

the theorems obtained in this paper are as follows:

Theorem I. If $f(x, y) \in L^{p}, p>1$, then the double Fourier series (1.2) is strongly summable to $s$ for every positive index $k$ whenever ${ }^{3}$

$$
\Phi_{x, v}^{(p)}(u, v)=o(u v) .
$$

THEOREM II. If $f(x, y) \in L^{p}, p>1$, then the Fourier series of $f(x, y)$ is strongly summable almost everywhere to $f(x, y)$ for every positive index $k$.

The question whether the hypothesis in Theorem II may be replaced by $f \log ^{+}|f| \in L$ is unsettled in this note. Corresponding questions in Fourier series of a single variable have been answered affirmatively by Marcinkiewicz [6] and Zygmund [12]. Indeed, the theorem holds under the weaker hypothesis $f \in L$. We content ourselves with establishing the following theorem.

\footnotetext{
$=0$.

${ }^{3}$ We use the symbol $o(u v)$ to denote a function of $u$ and $v$ such that $\lim _{u, v \rightarrow 0} o(u v) / u v$
} 
Theorem III. If $f(x, y) \log ^{+}|f(x, y)| \in L$ and $\sigma_{m, n}(x, y)$ denotes the $(m, n)$ th Fejer sum of the Fourier series of $f(x, y)$, then the relation

$$
\lim _{m, n \rightarrow \infty} \frac{1}{(m+1)(n+1)} \sum_{\mu=0}^{m} \sum_{\nu=0}^{n}\left|\sigma_{\mu, \nu}(x, y)-f(x, y)\right|^{k}=0
$$

holds true almost everywhere, where $k>0$.

2. Lemmas. Before proving our theorems, we prove a number of lemmas:

Lemma 1. If $f(x, y) \in L^{p}, p>1$, then

$$
\lim _{h, k \rightarrow 0} \frac{1}{h k} \int_{x_{0}}^{x_{0}+h} \int_{y_{0}}^{y_{0}+k} f(x, y) d x d y=f\left(x_{0}, y_{0}\right)
$$

at almost every point $\left(x_{0}, y_{0}\right)$.

This theorem is due to Zygmund [11]. Compare also [5] and [9].

Lemma 2. If $f(x, y) \in L^{p}, p>1$, then at almost every point $(x, y)$,

$$
\int_{0}^{h} \int_{0}^{k}|f(x \pm u, y \pm v)-f(x, y)|^{p} d u d v=o(h k)
$$

as $h, k \rightarrow 0$.

Proof. Let $\alpha$ be a rational number, and $E_{\alpha}$ the set of points $(x, y)$ such that

$$
\frac{1}{h k} \int_{0}^{h} \int_{0}^{k}|f(x \pm u, y \pm v)-\alpha|^{p} d u d v
$$

does not tend to $|f(x, y)-\alpha|^{p}$ as $h, k \rightarrow 0$. In virtue of Lemma $1, E_{\alpha}$ is of measure zero, and so also is the sum $E$ of all $E_{\alpha}$. Let $(x, y)$ be not a point of $E$ and let $\beta$ be a rational number, then, by Minkowski's inequality,

$$
\begin{aligned}
\left\{\frac{1}{h k} \int_{0}^{h} \int_{0}^{k} \mid f(x \pm\right. & u, y \pm v)-f(x, y) \mid p d u d v\}^{1 / p} \\
\leqq & \left\{\frac{1}{h k} \int_{0}^{h} \int_{0}^{k}|f(x \pm u, y \pm v)-\beta|^{p} d u d v\right\}^{1 / p} \\
& +\left\{\frac{1}{h k} \int_{0}^{h} \int_{0}^{k}|\beta-f(x, y)|^{p} d u d v\right\}^{1 / p}
\end{aligned}
$$

which tends to $2|f(x, y)-\beta|$ as $h, k \rightarrow 0$. As $\beta \rightarrow f(x, y)$, the result follows. 
Lemma 3. Let $f(x, y) \in L^{p}, 1<p \leqq 2,1 / p+1 / q=1$, and let the Fourier series of $f(x, y)$ be given in the complex form:

then

$$
f(x, y) \sim \sum_{\mu=-\infty}^{\infty} \sum_{\nu=-\infty}^{\infty} c_{\mu, \nu} e^{i(\mu x+\nu v)}
$$

(2.1) $\left\{\sum_{\mu=-\infty}^{\infty} \sum_{\nu=-\infty}^{\infty}\left|c_{\mu, \nu}\right|^{q}\right\}^{1 / q} \leqq\left\{\frac{1}{4 \pi^{2}} \int_{-\pi}^{\pi} \int_{-\pi}^{\pi}|f(x, y)| p d x d y\right\}^{1 / p}$.

This is a double series analogue of the Young-Hausdorff theorem, and may be proved by the method of M. Riesz ${ }^{4}$ with an obvious modification.

We also require the following formula of integration by parts:

$$
\begin{aligned}
& \int_{a_{1}}^{a_{2}} d u \int_{b_{1}}^{b_{2}} \rho \psi^{\prime}(u) \psi^{\prime \prime}(v) d u d v \\
&=\rho_{1}\left(a_{2}, b_{2}\right) \psi\left(a_{2}, b_{2}\right)-\int_{a_{1}}^{a_{2}} \rho_{1}\left(u, b_{2}\right) \psi_{u}\left(u, b_{2}\right) d u \\
&-\int_{b_{1}}^{b_{2}} \rho_{1}\left(a_{2}, v\right) \psi_{v}\left(a_{2}, v\right) d v+\int_{a_{1}}^{a_{2}} d u \int_{b_{1}}^{b_{2}} \rho_{1} \psi_{u v} d v
\end{aligned}
$$

where

$$
\psi(u, v)=\psi^{\prime}(u) \psi^{\prime \prime}(v), \quad \rho_{1}(u, v)=\int_{a_{1}}^{u} d \sigma \int_{b_{1}}^{v} \rho(\sigma, t) d t .
$$

This formula is valid if $\rho$ is integrable on $\left(a_{1}, b_{1} ; a_{2}, b_{2}\right), \psi^{\prime}$ is absolutely continuous on $\left(a_{1}, a_{2}\right)$, and $\psi^{\prime \prime}$ is absolutely continuous on $\left(b_{1}, b_{2}\right)$.

3. Proof of Theorem I. Without loss of generality, we may assume that $x=0, y=0$. So that

$$
s_{m, n}=\frac{1}{\pi^{2}} \int_{0}^{\pi} \int_{0}^{\pi} f(u, v) \frac{\sin (m+1 / 2) u}{\sin u / 2} \frac{\sin (n+1 / 2) v}{\sin v / 2} d u d v .
$$

We have to deduce

$$
\sum_{\mu=0}^{m} \sum_{\nu=0}^{n}\left|s_{\mu, \nu}-s\right|^{k}=o(u v)
$$

from (1.3).

Write

${ }^{4}$ M. Riesz [7], see also A. Zygmund [13]. 


$$
\begin{aligned}
\pi^{2}\left(s_{\mu, \nu}-s\right)= & \int_{0}^{\pi} \int_{0}^{\pi} \phi(u, v) \frac{\sin (m+1 / 2) u}{\sin u / 2} \frac{\sin (n+1 / 2) v}{\sin v / 2} d u d v \\
= & \int_{0}^{\pi} \int_{0}^{\pi} \phi(u, v)\left(\sin \mu u \cot \frac{u}{2} \sin \nu v+\sin \mu u \cot \frac{u}{2} \cos \nu v\right. \\
& \left.+\cos \mu u \sin \nu v \cot \frac{v}{2}+\cos \mu u \cos \nu v\right) d u d v \\
= & I_{1}(\mu, \nu)+I_{2}(\mu, \nu)+I_{3}(\mu, \nu)+I_{4}(\mu, \nu),
\end{aligned}
$$

and for $0<\mu \leqq m, 0<\nu \leqq n$,

$$
\begin{aligned}
I_{i}(\mu, \nu)= & \int_{0}^{m^{-1}} \int_{0}^{n^{-1}}+\int_{0}^{m^{-1}} \int_{n^{-1}}^{\pi}+\int_{m^{-1}}^{\pi} \int_{0}^{n^{-1}}+\int_{m^{-1}}^{\pi} \int_{n^{-1}}^{\pi} \\
= & I_{i 1}(\mu, \nu ; m, n)+I_{i 2}(\mu, \nu ; m, n) \\
& +I_{i 3}(\mu, \nu ; m, n)+I_{i 4}(\mu, \nu ; m, n),
\end{aligned}
$$

where $i=1,2,3$. For brevity, we also write $I_{i j}(\mu, \nu)$ for $I_{i j}(\mu, \nu ; m, n)$. Accordingly,

$$
\pi^{2}\left(s_{\mu, \nu}-s\right)=\sum_{i=1}^{3} \sum_{j=1}^{4} I_{i j}(\mu, \nu)+I_{4}(\mu, \nu) .
$$

It follows from Minkowski's inequality that

$$
\begin{aligned}
\pi^{2}\left\{\sum_{\mu=0}^{m} \sum_{\nu=0}^{n}\left|s_{\mu, \nu}-s\right| k\right\}^{1 / k} \leqq & \sum_{i=1}^{3} \sum_{j=1}^{4}\left\{\sum_{\mu=0}^{m} \sum_{\nu=0}^{n}\left|I_{i j}(\mu, \nu)\right|^{k}\right\}^{1 / k} \\
& +\left\{\sum_{\mu=0}^{m} \sum_{\nu=0}^{n}\left|I_{4}(\mu, \nu)\right|^{k}\right\}^{1 / k} .
\end{aligned}
$$

In the first place, by the analogue of the Riemann-Lebesgue theorem $^{5} I_{4}(\mu, \nu)$ tends to zero as $\mu, \nu \rightarrow \infty$. Hence

$$
\left\{\sum_{\mu=0}^{m} \sum_{\nu=0}^{n}\left|I_{4}(\mu, \nu)\right| k\right\}^{1 / k}=o(m n)^{1 / k} .
$$

Secondly, let us consider the integrals $I_{11}, I_{21}$ and $I_{31}$. Write

$$
K(u, v) \equiv K(u, v ; \mu, \nu)=\sin \mu u \cot u / 2 \sin \nu v \cot v / 2,
$$

then for $0<u \leqq \pi$ and $0<v \leqq \pi$ there is a constant $A$ such that

$$
u v \max \left(|K|, \mu^{-1}\left|K_{u}\right|, \nu^{-1}\left|K_{v}\right|, \mu^{-1} \nu^{-1}\left|K_{u v}\right|\right) \leqq A .
$$

We also write

${ }^{5}$ W. H. Young $[10$, p. 138]. 


$$
\Phi(u, v)=\int_{0}^{u} \int_{0}^{v} \phi(\xi, \eta) d \xi d \eta,
$$

which is $o(u v)$ by (1.3). Then on applying (2.2),

$$
\begin{aligned}
I_{11}(\mu, \nu)= & \int_{0}^{m^{-1}} \int_{0}^{n^{-1}} \phi(u, v) K(u, v ; \mu, \nu) d u d v \\
= & \Phi\left(m^{-1}, n^{-1}\right) K\left(m^{-1}, n^{-1}\right) \\
& -\int_{0}^{m^{-1}} \Phi\left(u, n^{-1}\right) K_{u}\left(u, n^{-1}\right) d u \\
& -\int_{0}^{n^{-1}} \Phi\left(m^{-1}, v\right) K_{v}\left(m^{-1}, v\right) d v \\
& +\int_{0}^{m^{-1}} d u \int_{0}^{n^{-1}} \Phi(u, v) K_{u v} d v .
\end{aligned}
$$

Since $0<\mu \leqq m, 0<\nu \leqq n$, it is easily seen from (3.4) and (3.5) that

$$
I_{11}(\mu, \nu)=o(1) \text {. }
$$

In a similar manner, we can prove $I_{21}(\mu, \nu)=o(1), I_{31}(\mu, \nu)=o(1)$. Hence we obtain

$$
\left\{\sum_{\mu=0}^{m} \sum_{\nu=0}^{n}\left|I_{i 1}(\mu, \nu)\right|^{k}\right\}^{1 / k}=o(m n)^{1 / k} \quad(i=1,2,3) .
$$

Thirdly, we consider the integrals $I_{14}, I_{24}$ and $I_{34}$. We have

$$
\begin{aligned}
I_{14}(\mu, \nu)= & \int_{m^{-1}}^{\pi} \int_{n^{-1}}^{\pi} \phi(u, v) \sin \mu u \cot \frac{u}{2} \sin \nu v \cot \frac{v}{2} d u d v \\
= & \int_{m^{-1}}^{\pi} \sin \mu u \cot \frac{u}{2} d u \\
& \cdot \int_{n^{-1}}^{\pi} \cot \frac{v}{2}\left(\frac{\partial}{\partial v} \int_{0}^{v} \sin \nu y \phi(u, y) d y\right) d v \\
= & \int_{m^{-1}}^{\pi} \sin \mu u \cot \frac{u}{2} d u\left(-\cot \frac{1}{2 n} \int_{0}^{n^{-1}} \sin \nu y \phi(u, y) d y\right. \\
& \left.+\frac{1}{2} \int_{n^{-1}}^{\pi} \csc ^{2} \frac{v}{2} d v \int_{0}^{v} \sin \nu y \phi(u, y) d y\right) \\
= & I_{14}^{\prime}+I_{14}^{\prime \prime},
\end{aligned}
$$

say, where $I_{14}^{\prime}$ is equal to 


$$
\begin{aligned}
& -\cot \frac{1}{2 n} \int_{m^{-1}}^{\pi} \cot \frac{u}{2}\left(\frac{d}{d u} \int_{0}^{u} \int_{0}^{n^{-1}} \sin \mu x \sin \nu y \phi(x, y) d x d y\right) d u \\
= & \cot \frac{1}{2 m} \cot \frac{1}{2 n} \int_{0}^{m^{-1}} \int_{0}^{n^{-1}} \sin \mu x \sin \nu y \phi(x, y) d x d y \\
& -\frac{1}{2} \cot \frac{1}{2 n} \int_{m^{-1}}^{\pi} \csc ^{2} \frac{u}{2} d u \int_{0}^{u} \int_{0}^{n^{-1}} \sin \mu x \sin \nu y \phi(x, y) d x d y .
\end{aligned}
$$

Let $c_{\mu, \nu}(\alpha, \beta)$ denote the $(\mu, \nu)$ th Fourier coefficient of the odd-odd function $\chi(x, y)$ which is equal to $\phi(x, y)$ in the rectangle $(0, \alpha ; 0, \beta)$ and to zero elsewhere. Then we may write

$$
\begin{aligned}
I_{14}^{\prime}= & \frac{\pi^{2}}{4} \cot \frac{1}{2 m} \cot \frac{1}{2 n} c_{\mu, \nu}\left(\frac{1}{m}, \frac{1}{n}\right) \\
& -\frac{\pi^{2}}{8} \cot \frac{1}{2 n} \int_{m^{-1}}^{\pi} \csc ^{2} \frac{u}{2} c_{\mu, \nu}\left(u, \frac{1}{n}\right) d u,
\end{aligned}
$$

and $I_{14}^{\prime \prime}$ may be written as

$$
\begin{aligned}
& \frac{1}{2} \int_{m^{-1}}^{\pi} \cot \frac{u}{2} d u \\
& \quad \cdot \int_{n^{-1}}^{\pi} \csc ^{2} \frac{v}{2}\left(\frac{\partial}{\partial u} \int_{0}^{u} \int_{0}^{v} \sin \mu x \sin \nu y \phi(x, y) d x d y\right) d v \\
& =\frac{\pi^{2}}{8} \int_{m^{-1}}^{\pi} \cot \frac{u}{2}\left(\frac{\partial}{\partial u} \int_{n^{-1}}^{\pi} \csc ^{2} \frac{v}{2} c_{\mu, \nu}(u, v) d v\right) d u \\
& =-\frac{\pi^{2}}{8} \cot \frac{1}{2 m} \int_{n^{-1}}^{\pi} \csc ^{2} \frac{v}{2} c_{\mu, \nu}\left(\frac{1}{m}, v\right) d v \\
& \quad+\frac{\pi^{2}}{16} \int_{m^{-1}}^{\pi} \int_{n^{-1}}^{\pi} \csc ^{2} \frac{u}{2} \csc ^{2} \frac{v}{2} c_{\mu, \nu}(u, v) d u d v
\end{aligned}
$$

It follows from (3.8), (3.9) and (3.10) that

$$
\begin{aligned}
\left(\sum_{\mu=0}^{m} \sum_{\nu=0}^{n}\left|I_{14}(\mu, \nu)\right|^{k}\right)^{1 / k} & \leqq \\
\leqq & \cot \frac{1}{2 m} \cot \frac{1}{2 n}\left(\sum_{\mu=\nu}^{m} \sum_{\nu=0}^{n}\left|c_{\mu, \nu}\left(\frac{1}{m}, \frac{1}{n}\right)\right|^{k}\right)^{1 / k} \\
& +A \cot \frac{1}{2 n} \int_{m^{-1}}^{\pi} \csc ^{2} \frac{u}{2}\left(\sum_{\mu=0}^{m} \sum_{\nu=0}^{n}\left|c_{\mu, \nu}\left(u, \frac{1}{n}\right)\right|^{k}\right)^{1 / k} d u
\end{aligned}
$$




$$
\begin{gathered}
+A \cot \frac{1}{2 m} \int_{n^{-1}}^{\pi} \csc ^{2} \frac{v}{2}\left(\sum_{\mu=0}^{m} \sum_{\nu=0}^{n}\left|c_{\mu, \nu}\left(\frac{1}{m}, v\right)\right|^{k}\right)^{1 / k} d v \\
+A \cot \frac{1}{2 m} \cot \frac{1}{2 n} \int_{m^{-1}}^{\pi} \int_{n^{-1}}^{\pi} \csc ^{2} \frac{u}{2} \csc ^{2} \frac{v}{2} \\
\cdot\left(\sum_{\mu=0}^{m} \sum_{\nu=0}^{n}\left|c_{\mu, \nu}(u, v)\right|^{k}\right)^{1 / k} d u d v .
\end{gathered}
$$

Now we assume, without loss of generality, that $k>2, k^{\prime}=k /(k-1)$ $<p$, so that by Lemma 3,

$$
\begin{aligned}
\left(\sum_{\mu=0}^{m} \sum_{\nu=0}^{n}\left|c_{\mu, \nu}(u, v)\right|^{k}\right)^{1 / k} & \leqq\left(\frac{1}{4 \pi^{2}} \int_{-\pi}^{\pi} \int_{-\pi}^{\pi}|\chi(x, y)| k^{\prime} d x d y\right)^{1 / k^{\prime}} \\
& =\left(\frac{1}{4 \pi^{2}} \int_{-u}^{u} \int_{-v}^{v}|\phi(x, y)|^{k^{\prime}} d x d y\right)^{1 / k^{\prime}} \\
& =o(u v)^{1 / k^{\prime}}
\end{aligned}
$$

since the condition (1.3) is satisfied a fortiori when $p$ is replaced by the smaller index $k^{\prime}$. Therefore

$$
\begin{aligned}
\left(\sum_{\mu=0}^{m} \sum_{\nu=0}^{n}\left|I_{14}(\mu, \nu)\right|^{k}\right)^{1 / k} \leqq & A m n o(m n)^{-1 / k^{\prime}}+A n \int_{m^{-1}}^{\pi}\left(\frac{u}{n}\right)^{1 / k^{\prime}} \frac{d u}{u^{2}} \\
& +A m \int_{n^{-1}}^{\pi}\left(\frac{v}{m}\right)^{1 / k^{\prime}} \frac{d v}{v^{2}} \\
& +A \int_{m^{-1}}^{\pi} \int_{n^{-1}}^{\pi} \frac{(u v)^{1 / k^{\prime}}}{u^{2} v^{2}} d u d v=o(m n)^{1 / k}
\end{aligned}
$$

The integral $I_{24}(\mu, \nu)$ is equal to

$$
\begin{aligned}
\int_{m^{-1}}^{\pi} \cot \frac{u}{2}\left(\frac{d}{d u} \int_{0}^{u} d x \int_{n^{-1}}^{\pi} \phi(x, y) \sin \mu x \cos \nu y d y\right) d u \\
=\frac{\pi^{2}}{4} \cot \frac{1}{2 m}\left[c_{\mu, \nu}^{\prime}\left(\frac{1}{m}, \pi\right)-c_{\mu, \nu}^{\prime}\left(\frac{1}{m}, \frac{1}{n}\right)\right] \\
-\frac{\pi^{2}}{8} \int_{m^{-1}}^{\pi} \csc ^{2} \frac{u}{2}\left[c_{\mu, \nu}^{\prime}(u, \pi)-c_{\mu, \nu}^{\prime}\left(u, \frac{1}{n}\right)\right] d u,
\end{aligned}
$$

where the $c_{\mu, \nu}^{\prime}(\alpha, \beta)(\mu, \nu=0,1,2, \cdots)$ denote the Fourier coefficients of the odd-even function $\chi^{\prime}(x, y)$ which is equal to $\phi(x, y)$ in the rectangle $(0, \alpha ; 0, \beta)$ and to zero elsewhere. In virtue of Minkowski's inequality and Lemma 3 , it is easily seen that 


$$
\left(\sum_{\mu=0}^{m} \sum_{\nu=0}^{n}\left|I_{24}(\mu, \nu)\right|^{k}\right)^{1 / k}=o(m n)^{1 / k}
$$

The integral $I_{34}$ can be treated in the same manner as $I_{14}$. We omit the details. Collecting the above results, we obtain

$$
\left(\sum_{\mu=0}^{m} \sum_{\nu=0}^{n}\left|I_{i 4}(\mu, \nu)\right|^{k}\right)^{1 / k}=o(m n)^{1 / k} \quad(i=1,2,3) .
$$

Fourthly, we estimate the integrals $I_{12}, I_{22}$ and $I_{32}$. We have

$$
\begin{aligned}
I_{12}(\mu, \nu)= & \int_{0}^{m^{-1}} \int_{n^{-1}}^{\pi} \phi(u, v) \sin \mu u \cot \frac{u}{2} \sin \nu v \cot \frac{v}{2} d u d v \\
= & \int_{0}^{m^{-1}} \cot \frac{u}{2} \sin \mu u d u \\
& \cdot \int_{n^{-1}}^{\pi} \cot \frac{v}{2}\left[\frac{\partial}{\partial v} \int_{0}^{v} \phi(u, y) \sin \nu y d y\right] d v \\
= & \int_{0}^{m^{-1}} \cot \frac{u}{2} \sin \mu u\left\{-\frac{\pi}{2} \cot \frac{1}{2 n} c_{\nu}\left(u, \frac{1}{n}\right)\right. \\
& \left.+\frac{\pi}{4} \int_{n^{-1}}^{\pi} \csc ^{2} \frac{v}{2} c_{\nu}(u, v) d v\right\} d u,
\end{aligned}
$$

where $c_{\nu}(\alpha, \beta)$ denotes the $\nu$ th Fourier coefficient of the odd function $\psi(u, \beta)$ which is equal to $\phi(u, v)$ for $0 \leqq v \leqq \beta$ and to zero for $\beta<v<\pi$. It follows from Young-Hausdorff's inequality that

so that

$$
\begin{aligned}
\left(\sum_{\nu=1}^{n}\left|c_{\nu}(u, v)\right|^{k}\right)^{1 / k} & \leqq\left(\frac{1}{\pi} \int_{-\pi}^{\pi}|\psi(u, y)|^{k^{\prime}} d y\right)^{1 / k^{\prime}} \\
& =\left(\frac{1}{\pi} \int_{-v}^{v}|\phi(u, y)|^{k^{\prime}} d y\right)^{1 / k^{\prime}},
\end{aligned}
$$

$$
\begin{aligned}
& \left(\sum_{\nu=0}^{n}\left|I_{12}(\mu, \nu)\right|^{k}\right)^{1 / k} \\
& \leqq A \cot \frac{1}{2 n} \int_{0}^{m^{-1}} \mu\left(\int_{-n^{-1}}^{n^{-1}}|\phi(u, y)|^{k^{\prime}} d y\right)^{1 / k^{\prime}} d u \\
& \quad+A \int_{0}^{m^{-1}} \mu d u \int_{n^{-1}}^{\pi} \csc ^{2} \frac{v}{2}\left(\int_{-v}^{v}|\phi(u, y)|^{k^{\prime}} d y\right)^{1 / k^{\prime}} d v
\end{aligned}
$$

Hölder's inequality gives 


$$
\begin{aligned}
& \int_{0}^{m^{-1}}\left(\int_{-v}^{v}|\phi(u, y)| k^{\prime} d y\right)^{1 / k^{\prime}} d u \\
& \leqq m^{1 / k^{\prime}-1}\left(\int_{0}^{m^{-1}} \int_{-v}^{v}|\phi(u, y)|^{k^{\prime}} d y d u\right)^{1 / k^{\prime}}=m^{1 / k^{\prime}-1} o\left(\frac{v}{m}\right)^{1 / k^{\prime}}
\end{aligned}
$$

Hence (3.13) is reduced to

$$
\begin{aligned}
\left(\sum_{\nu=0}^{n}\left|I_{12}(\mu, \nu)\right|^{k}\right)^{1 / k} & \\
& \leqq \mu \cot \frac{1}{2 n} m^{1 / k^{\prime}-1} o(m n)^{-1 / k^{\prime}}+\mu m^{1 / k^{\prime}-1} \int_{n^{-1}}^{\pi} v^{-2} o\left(\frac{v}{m}\right)^{1 / k^{\prime}} d v \\
& =o(n)^{1 / k} .
\end{aligned}
$$

Thus we obtain $\left(\sum_{\mu=0}^{m} \sum_{\nu=0}^{n}\left|I_{12}(\mu, \nu)\right|^{k}\right)^{1 / k}=o(m n)^{1 / k}$. The integrals $I_{22}$ and $I_{32}$ may be treated in a similar manner as above. The following relations are thus established:

$$
\left(\sum_{\mu=0}^{m} \sum_{\nu=0}^{n}\left|I_{i 2}(\mu, \nu)\right|^{k}\right)^{1 / k}=o(m n)^{1 / k} \quad(i=1,2,3) .
$$

Finally, we have to consider the integrals $I_{13}, I_{23}$ and $I_{33}$. The discussion of $I_{13}$ is the same as $I_{12}$, and the integral $I_{23}$ has been treated implicitly in the discussion of $I_{24}$. It remains therefore only to deal with $I_{33}$. Regard the integrals

$$
\begin{aligned}
& I_{33}(\mu, \nu)=\int_{m^{-1}}^{\pi} \cos \mu u d u \int_{0}^{n^{-1}} \phi(u, v) \sin \nu v \cot \frac{v}{2} d v \\
&(\mu=0,1,2, \cdots)
\end{aligned}
$$

as the Fourier coefficients of the function of $u$ which is equal to

$$
\int_{0}^{n^{-1}} \phi(u, v) \sin \nu v \cot \frac{v}{2} d v \quad \text { for } m^{-1} \leqq u \leqq \pi,
$$

and to zero for $-\pi \leqq u<m^{-1}$, then by Hausdorff's inequality,

$$
\begin{aligned}
\left\{\sum_{\mu=0}^{m}\left|I_{33}(\mu, \nu)\right| k\right\}^{1 / k} & \leqq \\
& \left.\leqq \frac{1}{4 \pi^{2}} \int_{m^{-1}}^{\pi}\left|\int_{0}^{n^{-1}} \sin \nu v \cot \frac{v}{2} \phi(u, v) d v\right|^{k^{\prime}} d u\right)^{1 / k} \\
& \leqq A_{\nu}\left(\int_{m^{-1}}^{\pi}\left(\int_{0}^{n^{-1}}|\phi(u, v)| d v\right)^{k^{\prime}} d u\right)^{1 / k^{\prime}}
\end{aligned}
$$


It follows from Hölder's inequality that

$$
\int_{0}^{n^{-1}}|\phi(u, v)| d v \leqq n^{1 / k^{\prime}-1}\left(\int_{0}^{n}|\phi(u, v)| k^{\prime} d v\right)^{1 / k^{\prime}}
$$

so that

$$
\nu n^{1 / k^{\prime}-1}\left(\int_{m^{-1}}^{\pi} d u \int_{0}^{n^{-1}}|\phi(u, v)| k^{\prime} d v\right)^{1 / k^{\prime}}=\nu n^{1 / k^{\prime}-1} O\left(n^{-1 / k^{\prime}}\right)=O(1) .
$$

Hence from (3.15) it results that $\left\{\sum_{\mu=0}^{m} \sum_{\nu=0}^{n}\left|I_{33}(\mu, \nu)\right|^{k}\right\}^{1 / k}=o(m n)^{1 / k}$. The following relations are thus proved:

$$
\left\{\sum_{\mu=0}^{m} \sum_{\nu=0}^{n}\left|I_{i 3}(\mu, \nu)\right|^{k}\right\}^{1 / k}=o(m n)^{1 / k} \quad(i=1,2,3) .
$$

Collecting the results (3.2), (3.3), (3.7), (3.11), (3.14), and (3.16) we obtain (3.1). Theorem I is thus proved.

4. Proof of Theorem II. On account of Theorem I, it suffices to show that the condition (1.3) is satisfied almost everywhere when $s=f(x, y)$. Observing

$$
\begin{aligned}
4\left|\phi_{x, y}(u, v)\right| \leqq & |f(x+u, y+v)-f(x, y)| \\
& +|f(x+u, y-v)-f(x, y)| \\
& +|f(x-u, y+v)-f(x, y)| \\
& +|f(x-u, y-v)-f(x, y)|,
\end{aligned}
$$

and employing Minkowski's inequality, we immediately obtain the desired result from Lemma 2.

5. Proof of Theorem III. The proof depends upon the following two lemmas:

LEMMA 4. Theorem III holds good when $f(x, y)$ is bounded.

Since a bounded function belongs to $L^{p}, p>1$, the lemma follows from Theorem II.

Lemma 5. Let $h(x)$ be a function such that $h \log ^{+}|h| \in L(-\pi, \pi)$. Let $\beta_{m}=\beta_{m}(x, h)(m=0,1,2, \cdots)$ be the Fejerr sums of the Fourier series of $h(x)$, and $\beta^{*}(x)=\sup _{m}\left|\beta_{m}(x)\right|$, then

$$
\int_{-\pi}^{\pi} \beta^{*}(x) d x \leqq A \int_{-\pi}^{\pi}|h| \log ^{+}|h| d x+B,
$$

where $A$ and $B$ are absolute constants. 
This lemma is due to Hardy and Littlewood [4]. See also [13, p. 248].

Before proving the theorem, we extend Lemma 5 to the case of two variables. Let, for fixed $y$,

$$
g(x, y)=\sup _{m} \beta_{m}(x ;|f|) .
$$

Integrating this equation with respect to $y$, we obtain

$$
\begin{aligned}
& \int_{-\pi}^{\pi} \int_{-\pi}^{\pi} g(x, y) d x d y \\
& \quad \leqq 2 \pi A \int_{-\pi}^{\pi} \int_{-\pi}^{\pi}|f(x, y)| \log ^{+}|f(x, y)| d x d y+2 \pi B .
\end{aligned}
$$

Writing $K_{n}(x)$ for the Fejér kernel, we have

$$
\sigma_{m, n}(x, y ; f)=\frac{1}{\pi^{2}} \int_{-\pi}^{\pi} \int_{-\pi}^{\pi} f(u, v) K_{m}(x-u) K_{n}(y-v) d u d v .
$$

It follows that

$$
\left|\sigma_{m, n}(x, y ; f)\right| \leqq \frac{1}{\pi} \int_{-\pi}^{\pi} K_{n}(y-v) g(x, v) d v .
$$

In virtue of Lebesgue's theorem, the last expression tends to $g(x, y)$ at almost every point $(x, y)$. Therefore the relation

$$
\sigma^{*}(x, y ; f)=\limsup _{m, n \rightarrow \infty}\left|\sigma_{m, n}(x, y)\right| \leqq g(x, y)
$$

holds good almost everywhere. Combining this result with (5.1) we obtain

$$
\begin{aligned}
& \int_{-\pi}^{\pi} \int_{-\pi}^{\pi} \sigma^{*}(x, y ; f) d x d y \\
& \quad \leqq 2 \pi A \int_{-\pi}^{\pi} \int_{-\pi}^{\pi}|f(x, y)| \log ^{+}|f(x, y)| d x d y+2 \pi B
\end{aligned}
$$

Let $\lambda$ be a positive constant. Substituting $\lambda f$ for $f$ in (5.2), we obtain

$$
\begin{aligned}
& \int_{-\pi}^{\pi} \int_{-\pi}^{\pi} \sigma^{*}(x, y ; f) d x d y \\
& \quad \leqq 2 \pi A \int_{-\pi}^{\pi} \int_{-\pi}^{\pi}|f(x, y)| \log ^{+}|f(x, y)| d x d y+2 \pi \frac{B}{\lambda} .
\end{aligned}
$$


Let $\epsilon$ be a positive number; we take $\lambda$ so large that $2 \pi B / \lambda<\epsilon / 2$. Let

$$
f(x, y)=f^{\prime}(x, y)+f^{\prime \prime}(x, y)
$$

be such that $f^{\prime}$ is bounded;

$$
\int_{-\pi}^{\pi} \int_{-\pi}^{\pi}\left|f^{\prime \prime}(x, y)\right| d x d y<\epsilon
$$

$$
2 \pi A \int_{-\pi}^{\pi} \int_{-\pi}^{\pi}\left|f^{\prime \prime}(x, y)\right| \log ^{+}\left|\lambda f^{\prime \prime}(x, y)\right| d x d y+2 \pi \frac{B}{\lambda}
$$

Applying the inequality (5.3) to the function $f^{\prime \prime}(x, y)$, we obtain

$$
\int_{-\pi}^{\pi} \int_{-\pi}^{\pi} \sigma^{*}\left(x, y ; f^{\prime \prime}\right) d x d y<\epsilon
$$

by observing (5.5). Combining this relation with (5.4), we see that the set $E(\epsilon)$ of points $(x, y)$ such that either $\left|f^{\prime \prime}(x, y)\right|>\epsilon^{1 / 2}$ or $\sigma^{*}(x, y ; f)>\epsilon^{1 / 2}$ is of plane measure less than $2 \epsilon^{1 / 2}$. Now let $\sigma_{\mu, \nu}^{\prime}$ and $\sigma_{\mu, \nu}^{\prime \prime}$ denote respectively the $(\mu, \nu)$ th Fejér sums of the Fourier series of $f^{\prime}$ and $f^{\prime \prime}$, then

$$
\begin{aligned}
\left(\sum_{\mu=0}^{m} \sum_{\nu=0}^{n}\left|\sigma_{\mu, \nu}-f\right|^{k}\right) \leqq & \left(\sum_{\mu=0}^{m} \sum_{\nu=0}^{n}\left|\sigma_{\mu, \nu}^{\prime}-f\right|^{k}\right)^{1 / k} \\
& +\left(\sum_{\mu=0}^{m} \sum_{\nu=0}^{n}\left|\sigma_{\mu, \nu}^{\prime \prime}-f^{\prime \prime}\right|^{k}\right)^{1 / k} .
\end{aligned}
$$

The first term on the right-hand side is $o(m n)^{1 / k}$ almost everywhere, by Lemma 4 . And

$$
\begin{aligned}
\left(\sum_{\mu=0}^{m} \sum_{\nu=0}^{n}\left|\sigma_{\mu, \nu}^{\prime \prime}-f^{\prime \prime}\right| k\right)^{1 / k} & \\
& \leqq\left(\sum_{\mu=0}^{m} \sum_{\nu=0}^{n}\left|\sigma_{\mu, \nu}^{\prime \prime}\right|^{k}\right)^{1 / k}+\left(\sum_{\mu=0}^{m} \sum_{\nu=0}^{n}\left|f^{\prime \prime}\right|^{k}\right)^{1 / k} \\
& \leqq[(m+1)(n+1)]^{1 / k}\left(\sigma^{*}\left(x, y ; f^{\prime \prime}\right)+\left|f^{\prime \prime}\right|\right) .
\end{aligned}
$$

Hence, outside the set $E(\epsilon)$,

$$
\begin{aligned}
\limsup _{m, n \rightarrow \infty}\left\{\frac{1}{(m+1)(n+1)} \sum_{\mu=0}^{m} \sum_{\nu=0}^{n}\left|\sigma_{\mu, \nu}-f\right|^{k}\right\}^{1 / k} & \\
& \leqq \sigma^{*}\left(x, y ; f^{\prime \prime}\right)+\left|f^{\prime \prime}\right| \leqq 2 \epsilon^{1 / 2} .
\end{aligned}
$$

Since $\epsilon$ is arbitrary, the theorem follows. 


\section{BIBLIOGRAPHY}

1. G. H. Hardy and J. E. Littlewood, Sur la series de Fourier d'une fonction a carré sommable, C. R. Acad. Sci. Paris vol. 156 (1913) pp. 1307-1309.

2. - Notes on the theory of series (IV): On the strong summability of Fourier series, Proc. London Math. Soc. (2) vol. 26 (1927) pp. 273-286.

3. - The strong summability of Fourier series, Fund. Math. vol. 25 (1935) pp. $162-189$.

4. - A maximal theorem with function-theoretic applications, Acta Math. vol. 54 (1931) pp. 81-116.

5. B. Jessen, J. Marcinkiewicz and A. Zygmund, Note on the differentiability of multiple integrals, Fund. Math. vol. 25 (1935) pp. 217-234.

6. J. Marcinkiewicz, Sur la sommabilitê forte de sêries de Fourier, J. London Math. Soc. vol. 14 (1939) pp. 162-168.

7. M. Riesz, Sur les maxima des formes billinéaires et sur les fonctionnelles linéaires, Acta Math. vol. 49 (1926) pp. 465-497.

8. S. Saks, Remark on the differentiability of the Lebesgue indefinite integral, Fund. Math. vol. 22 (1934) pp. 257-261.

9. - Theory of integrals, Monografje Matematyczne, Warsaw, 1937.

10. W. H. Young, Multiple Fourier series, Proc. London Math. Soc. (2) vol. 11 (1913) pp. 133-184.

11. A. Zygmund, On the differentiability of multiple integrals, Fund. Math. vol. 23 (1934) pp. 143-149.

12. - On the convergence and summability of power series on the circle of convergence (II), Proc. London Math. Soc. vol. 47 (1942) pp. 326-350.

13. - Trigonometrical series, Monografje Matematyczne, Warsaw, 1935. 